\title{
Quantification of Lovastatin Produced by Monascus purpureus
}

\author{
A. Seenivasan ${ }^{\text {a, \# }}$, Sathyanarayana N. Gummadi ${ }^{\text {b }}$, Tapobrata Panda ${ }^{\mathrm{a}, *}$ and Thomas Théodore ${ }^{\mathrm{c}}$ \\ ${ }^{a}$ Biochemical Engineering Laboratory, Department of Chemical Engineering, Indian Institute of Technology, Madras, \\ Chennai 600036, Tamil Nadu, India \\ ${ }^{b}$ Department of Biotechnology, Indian Institute of Technology, Madras, Chennai-600036, Tamil Nadu, India \\ ${ }^{c}$ Department of Chemical Engineering, Siddaganga Institute of Technology, Tumkur - 572103, Karnataka, India \\ ${ }^{\#}$ Present address: Department of Chemical Engineering, SSN College of Engineering, Chennai-603110, Tamil Nadu, \\ India
}

\begin{abstract}
Development of a novel method for the quantification of lovastatin is an interesting problem in the analytical field. In the literature, many reports use spectrophotometric method for the quantification of lovastatin. However, the analysis of fermentation broth containing lovastatin appears to be inaccurate using spectrophotometric method. Hence, the estimation of lovastatin produced by Monascus purpureus and pure lovastatin was attempted by UV-visible spectrophotometer as well as HPLC. It was observed that the analogues and/or intermediates of lovastatin synthesized in the fermentation broth and the products of fermentation caused superimposition effect on the absorption spectrum. Phosphate is a medium constituent for the production of lovastatin by the organism which contributed significantly to the superimposition of absorption spectrum. On the other hand, HPLC analysis consistently gave reliable results for the estimation of lovastatin under all the experimental conditions studied.
\end{abstract}

Keywords: Lovastatin, Monascus purpureus, spectrophotometry, HPLC.

\section{INTRODUCTION}

The conversion of 3-hydroxy-3-methyl-glutaryl coenzyme A (HMG-CoA) into mevalonate is the rate- limiting step in the cholesterol cascade mediated by HMG-CoA reductase (E.C 1.1.1.88). Statins are structural analogues of HMG-CoA and bind with the enzyme at its active site. This alters the conformation of the enzyme. The reversible competitive binding between statin and the enzyme prevents the access of the substrate HMG-CoA to the HMG-CoA reductase [1-6]. Among all statins, lovastatin is the first approved by USFDA for the treatment of hypercholesterolemia in 1987. Lovastatin also serves as a precursor for other important statins like simvastatin and wuxistatin [7, 8].

Development of analytical methods for the estimation of lovastatin is an interesting topic due to the existing problems in the analytical techniques. Pure and fermentation-derived lovastatin were quantified mostly using UV-visible spectrophotometer [9-16], HPLC [17-20], and capillary electrophoresis [21, 22].

The spectrophotometric method of analysis of lovastatin is simple, faster, eco-friendly, and less laborious method of analysis than other analytical techniques. However, the spectrophotometric quantification of lovastatin is not

\footnotetext{
*Address correspondence to this author at the Department of Chemical Engineering, Indian Institute of Technology, Madras, Chennai 600036, Tamil Nadu, India; Tel: +91-44-2257 4160; E-mail: panda@iitm.ac.in
}

accurate in fermentation broth as well as for the system having competitive molecules which also absorbs light at $238 \mathrm{~nm}[2,3]$. Lovastatin has been produced commercially through fermentation route only $[1,6]$. Hence, it is important to measure the content of lovastatin accurately in the fermentation broth. Many researchers have used HPLC technique for the quantification of lovastatin in biological samples, but the reason for shifting/moving from the UVvisible spectrophotometer to HPLC has not been explained so far for the estimation of fermentation-derived lovastatin. In the present study, we have used UV-visible spectrophotometer and HPLC techniques for the quantification of lovastatin produced in the fermentation broth. Our objective was to highlight the various forms of interferences in the quantification of lovastatin with necessary experimental proof, which has not been reported so far.

\section{EXPERIMENTAL}

\subsection{Chemicals}

Lovastatin (lactone form) was procured from SigmaAldrich. All other chemicals were of analytical grade and procured from Sisco Research Laboratories Pvt. Ltd., Mumbai, India. For the assay and preparation of reagents, Milli-Q water was used throughout the study. Lovastatin was dissolved in acetonitrile to get a stock solution of $1 \mathrm{mg} / \mathrm{mL}$. The hydroxy acid form of lovastatin was prepared by hydrolysis as described by Yang and Hwang [23]. 


\subsection{Organism and Culture Conditions}

Monascus purpureus MTCC 369 was obtained from the Institute of Microbial Technology, Chandigarh, India. A suspension containing $10^{5}-10^{6}$ spores $/ \mathrm{mL}$ was used to inoculate a basal medium having the following composition (in g/L): dextrose, 100; peptone, $10 ; \mathrm{KNO}_{3}, 2 ; \mathrm{NH}_{4}\left(\mathrm{H}_{2} \mathrm{PO}_{4}\right)$, $2 ; \mathrm{MgSO}_{4} \cdot 7 \mathrm{H}_{2} \mathrm{O}, 0.5 ; \mathrm{CaCl}_{2}, 0.1$. The $\mathrm{pH}$ was adjusted to 6 using $0.1 \mathrm{~N} \mathrm{NaOH}$ solutions [16]. The culture was incubated at $30{ }^{\circ} \mathrm{C}$ for $48 \mathrm{~h}$ in an orbital shaker maintained at $120 \mathrm{rpm}$.

The defined production medium had the following composition (in g/L): dextrose, 29.59; $\mathrm{NH}_{4} \mathrm{Cl}, 3.86$; $\mathrm{KH}_{2} \mathrm{PO}_{4}, 1.73 ; \mathrm{MgSO}_{4} \cdot 7 \mathrm{H}_{2} \mathrm{O}, 0.86 ; \mathrm{MnSO}_{4} \cdot \mathrm{H}_{2} \mathrm{O}, 0.19$. The $\mathrm{pH}$ was adjusted to 6 [16]. The complex production medium contained (in g/L): rice powder, 34.4; peptone, 10.8; glycerol, 33.2; glucose, $129.2 ; \mathrm{MgSO}_{4} \cdot 7 \mathrm{H}_{2} \mathrm{O}, 1 ; \mathrm{KNO}_{3}, 2$. The $\mathrm{pH}$ was adjusted to 6 [24]. A 500-mL Erlenmeyer flask containing $100 \mathrm{~mL}$ of sterile medium was inoculated with $10 \%(\mathrm{v} / \mathrm{v})$ seed culture and incubated at $30^{\circ} \mathrm{C}$ for 15 days on a rotary shaker maintained at $120 \mathrm{rpm}$ [18]. Care was taken to restrict evaporation loss during fermentation. Suitable correction factor has been used in this regard.

\subsection{Analysis of Products}

\subsubsection{Pure Lovastatin}

Working standards of lactone- and hydroxy acid- forms of lovastatin were prepared and the absorbance was read at $238 \mathrm{~nm}$ using a UV-visible spectrophotometer (JASCO V630, Essex, UK). The zero-order absorption spectra were obtained over the wavelength range of 200-600 $\mathrm{nm}$ using the appropriate dispersion medium in a quartz cuvette $(1 \mathrm{~cm}$ path length) at $1.5 \mathrm{~nm}$ slit width $(\Delta \lambda)$.

The limit of quantification (QL) and detection limit (DL) was found using the equation given below,

$$
D L ; Q L=\frac{F \times(S D)}{a}
$$

where $\mathrm{F}$ value for $\mathrm{DL}$ and $\mathrm{QL}$ are $3.3 \mu \mathrm{g}$ and $10 \mu \mathrm{g}$, respectively. SD is the standard deviation of the blank and ' $a$ ' is the slope of the standard plot [25].

Quantitative HPLC was performed using a gradient HPLC (Shimadzu, Prominence HPLC, Kyoto, Japan) equipped with a photodiode array detector and Luna C18 column $(250 \times 4.6 \mathrm{~mm}, 5 \mu \mathrm{m})$. The mobile phase was composed of acetonitrile and acidified water $\left(0.1 \% \mathrm{H}_{3} \mathrm{PO}_{4}\right)$ $(60: 40, \mathrm{v} / \mathrm{v})$ which was filtered through a $0.22-\mu \mathrm{m}$ membrane filter. The flow rate of solvent and column temperature was maintained at $1 \mathrm{~mL} / \mathrm{min}$ and $40{ }^{\circ} \mathrm{C}$, respectively. A $20 \mu \mathrm{L}$ sample was injected into the column for analysis. The working standards of lactone- and hydroxy acid- forms of lovastatin were analyzed by HPLC at $238 \mathrm{~nm}$.

\subsubsection{Lovastatin Produced by Monascus Purpureus}

The sample (cell suspension) was homogenized by ultrasonication for 20 min followed by extraction with an equal volume of ethyl acetate at $60{ }^{\circ} \mathrm{C}$ for $30 \mathrm{~min}$ with intermittent shaking. The upper organic layer was isolated from the aqueous layer and dried under vacuum in a rotary evaporator. The dry residue was resuspended in pure acetonitrile. This suspension was filtered through a $0.22-\mu \mathrm{m}$ nylon filter paper (Pall Trincor ${ }^{\circledR}$, USA) before analysis by UV-visible spectrophotometer and HPLC [17].

Thin layer chromatography (TLC) (using dichloromethane/ethyl acetate $(70: 30, \mathrm{v} / \mathrm{v}))$ was done to confirm the presence of lovastatin [26].

Inductively-coupled plasma optical emission spectrometer (ICP-OES, Perkin-Elmer Optima 5300 DV, Shelton, CT) was used to measure potassium $\left(\lambda_{\max }=766.5\right.$ $\mathrm{nm})$ and phosphorus $\left(\lambda_{\max }=213.62 \mathrm{~nm}\right)$ present in the suspension and also released during ultrasonication. The flow rates of the auxiliary, plasma, and nebulizer were maintained at $0.2,15$, and $0.8 \mathrm{~L} / \mathrm{min}$ and that of the sample at $1.5 \mathrm{~mL} / \mathrm{min}$, respectively. A radiation source of $40 \mathrm{MHz}$ and RF power of 1300 were used in the system. Potassium and phosphorus were measured using the axially-viewed plasma set-up.

\section{RESULT AND DISCUSSION}

\subsection{Characterization of Pure Lovastatin Using Separately UV-visible Spectrophotometer and HPLC}

Pure lovastatin has three different absorption maxima at 232, 238, and $247 \mathrm{~nm}$ in UV-visible spectrophotometry ( $c f$. Fig. 1A), which suggests better identification of lovastatin from other compounds. The characteristics of the peaks could be due to the presence of diene [27]. The absorption spectra of both lactone form and hydroxy acid form of lovastatin in a mixture appear similar ( $c f$. Fig. 1). The regression analysis of the individual forms of lovastatin and molar extinction coefficient $(\varepsilon)$ are given in Table $\mathbf{1}$. The molar extinction coefficients for the lactone and hydroxy acid forms of lovastatin are $4.89 \times 10^{4} \pm 21.8$ and $5.99 \times 10^{4} \pm$ $36 \mathrm{M}^{-1} \mathrm{~cm}^{-1}$, respectively. This is valid for the estimation range of lovastatin between $2 \mu \mathrm{g}$ and $20 \mu \mathrm{g}$. Values of DL and QL for lovastatin are $0.39 \mu \mathrm{g}$ and $1.16 \mu \mathrm{g}$, respectively.

Results from the spectrophotometric analysis gave the total amount of lovastatin (hydroxy acid plus lactone forms) rather than the amount of the individual forms of lovastatin, while distinct peaks were obtained for the hydroxy acid and lactone forms of lovastatin by HPLC technique ( $c f$. Fig. 1B). Hence, the individual forms of lovastatin cannot be distinguished in both pure and fermentation derived samples by UV-visible spectrophotometer. Therefore, the spectrophotometric method of quantification of lovastatin could lead to a confusion in clinical studies, since the individual forms of lovastatin have specific properties, viz., pharmacological activity, solubility, lipophilic nature, and transportation characteristics.

The lactone form of lovastatin is more lipophilic than the hydroxy acid form. For this reason, the lactone form is accepted by the hepatic cells. This behavior determines the bioavailability of lovastatin. In liver, the diffused lactone form of lovastatin is converted into hydrolyzed forms including the hydroxy acid form by cytochrome P450 3A4 [28]. Hence, the quantification of the individual forms of lovastatin as well as hydrolyzed/metabolized products of 
Table 1. Assay parameters of UV-visible spectrophotometric measurements.

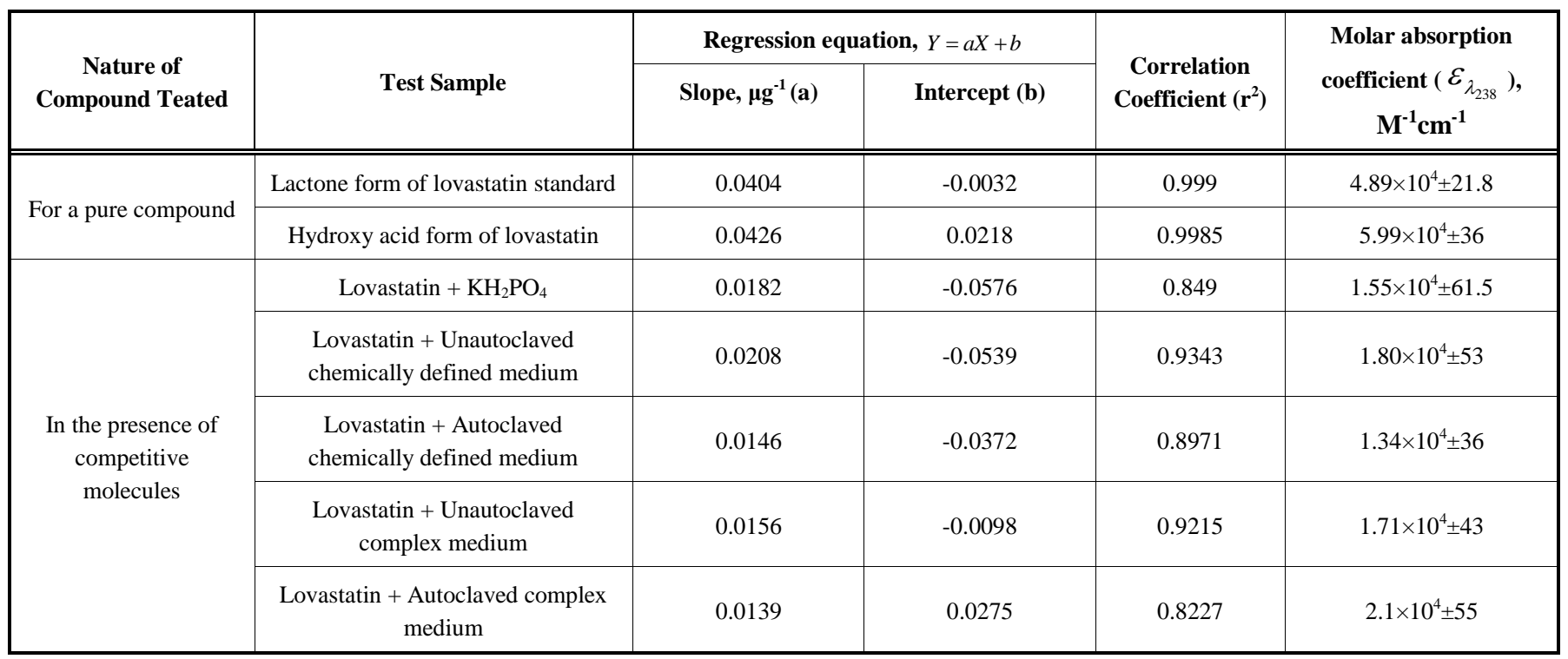

where $\mathrm{Y}$-is the absorbance value at $238 \mathrm{~nm}$ and $\mathrm{X}$ is the amount of lovastatin $(\mu \mathrm{g})$. The molar absorption coefficients are expressed as mean \pm standard deviation for the sample size of $\mathrm{n}=5$. The detection limit (DL) and quantification (DQ) are found $0.39 \mu \mathrm{g}$ and $1.16 \mu \mathrm{g}$, respectively from the equation, $D L ; Q L=\frac{F \times(S D)}{a}$, where F value for DL and QL are $3.3 \mu \mathrm{g}$ and $10 \mu \mathrm{g}$ and SD is the standard deviation of the blank. 'a' is the slope of the standard plot [25]. The regression model for HPLC data is $y=2 \times 10^{6} \mathrm{x}$ for all the cases (remained same), ' $\mathrm{y}$ ' is the detector response (AU) and ' $\mathrm{x}$ ' is the amount of lovastatin ( $\left.\mu \mathrm{g}\right)$.

lovastatin is necessary to determine the metabolic rate in controlled delivery systems. Several reports use the spectrophotometric method of quantification of in vitro and in vivo drug release characteristics [29-35]. A few studies have reported the controlled release characteristics of lovastatin in vitro by measuring lovastatin using UV-visible spectrophotometer and in vivo release characteristics of lovastatin measured by HPLC technique [29, 36].

\subsection{Effect of Phosphate and Potassium on Lovastatin Estimation}

The phosphate and potassium contents were estimated in the culture sample of $M$. purpureus grown in defined as well as in complex production medium, using ICP-OES. The samples were subjected to ultrasonication before estimation. Samples were collected every $24 \mathrm{~h}$ for 15 days. Significant amounts of phosphorus were released compared to
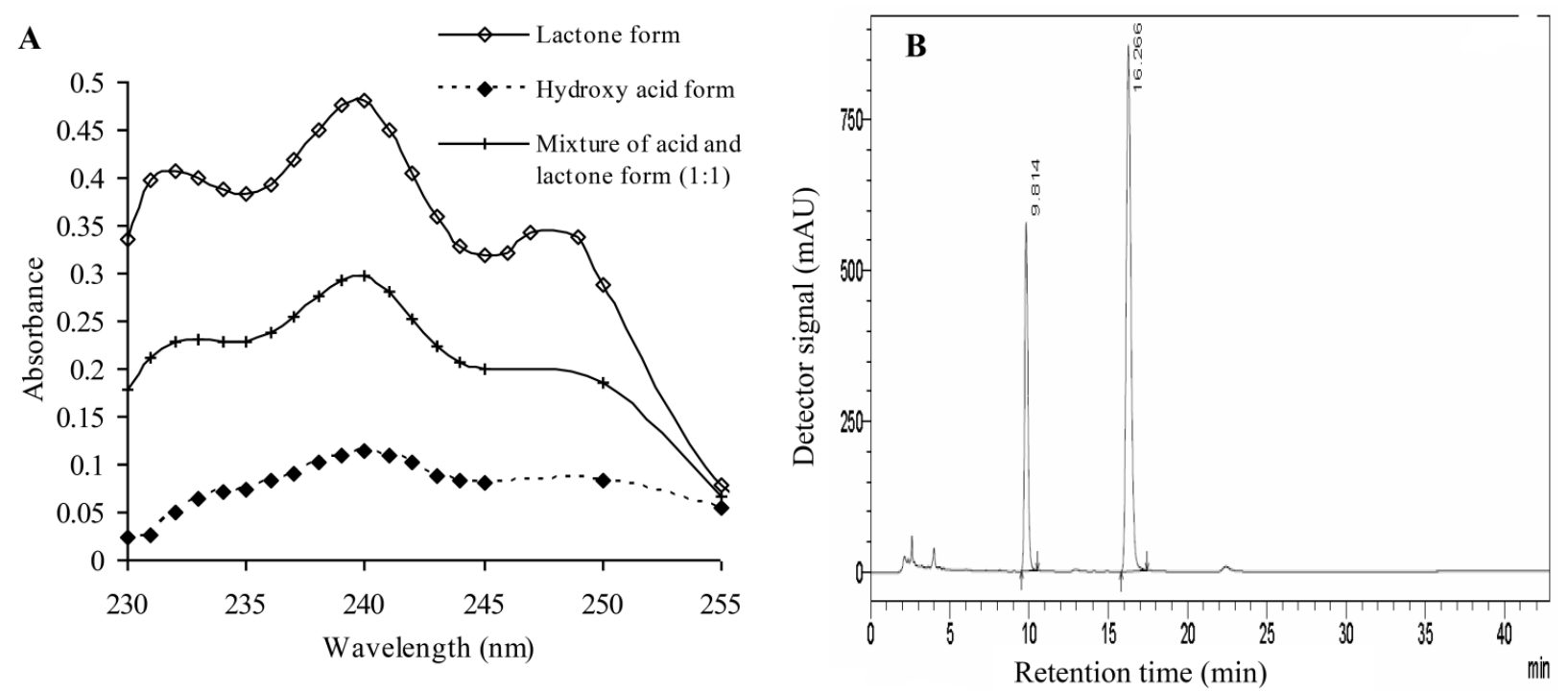

Fig. (1). UV-visible spectrophotometric and HPLC methods of estimation of pure lovastatin. (A) The lactone and hydroxy acid forms of lovastatin exhibited absorbance maxima $\left(\lambda_{\max }\right)$ at 232,238 , and $247 \mathrm{~nm}$ individually as well as in mixture (1:1) by UV-Visible spectrophotometer. (B) Both the forms of lovastatin quantified separately using HPLC. The retention times for hydroxy acid and lactone forms were 9.8 and $16.3 \mathrm{~min}$, respectively. 
potassium during the ultrasonication of the culture broth. The total amount of phosphorus in the defined medium was more than that in the complex medium. The maximum total amount of phosphorus (equivalent to $\mathrm{KH}_{2} \mathrm{PO}_{4}$ concentration) already present in the broth and released during ultrasonication was found to be $1.807 \mathrm{~g} / \mathrm{l}$ and $0.0175 \mathrm{~g} / \mathrm{l}$ for the defined and complex production medium, respectively. The effect of phosphate (equivalent to $\mathrm{KH}_{2} \mathrm{PO}_{4}$ concentration) in the estimation of a known quantity of pure lovastatin (lactone form) is shown in Figs. (2A) and (2C).

Jerotskaja et al. [37] measured some of the constituents in the spent human blood samples, after dialysis, using UVvisible spectrophotometer and quantified the levels of potassium and phosphate at 227 and $237 \mathrm{~nm}$, respectively. It
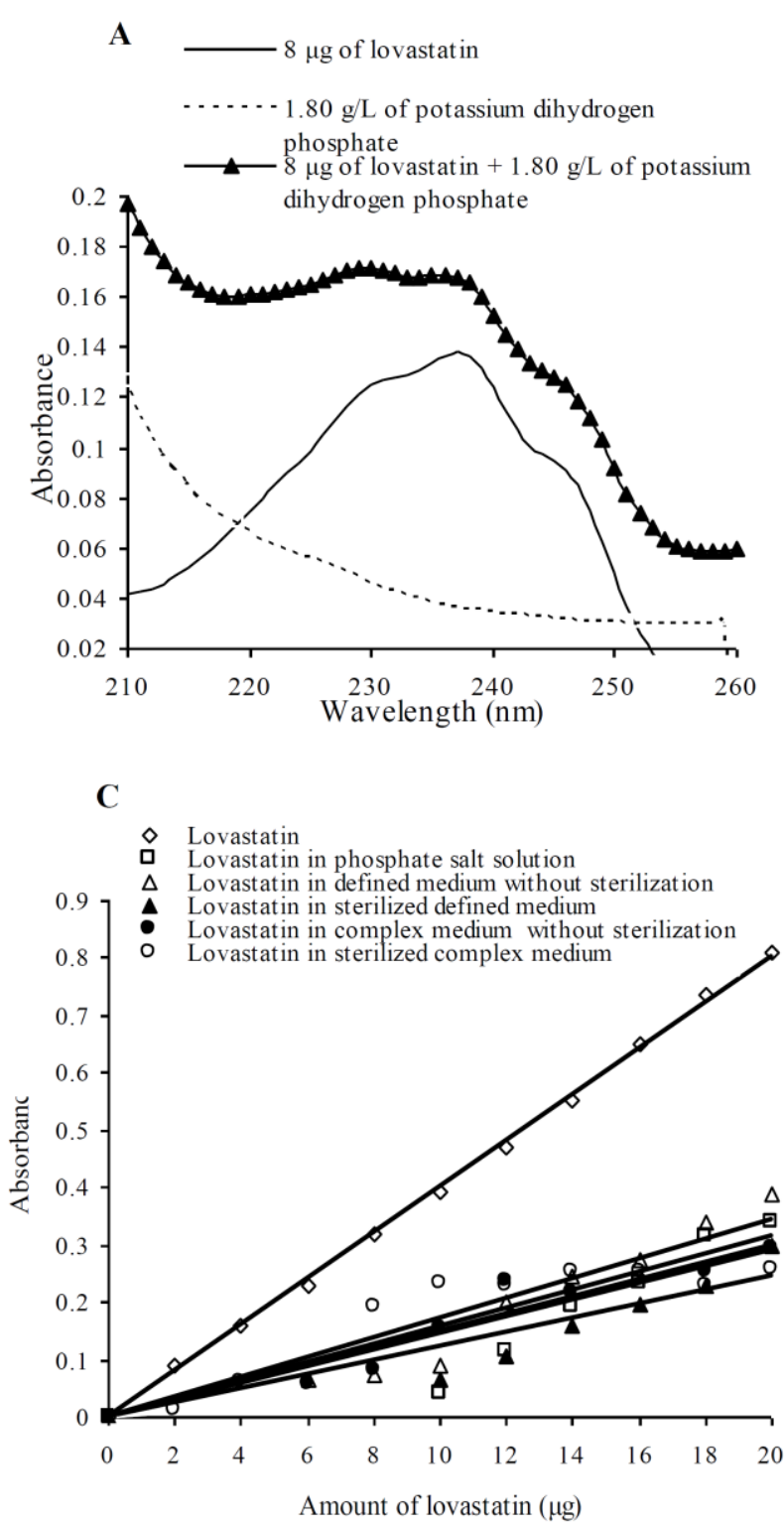

suggests that the phosphate and potassium already present in the medium as well as that released from cells after sonication led to errors in lovastatin estimation by UVvisible spectrophotometer. The presence of diene groups in the phosphate and its related compounds might absorb at 238 $\mathrm{nm}$. This could contribute to inaccurate measurement of this drug by the spectrophotometric method [27, 38].

It is pertinent to mention here that this study is relevant to a problem associated in the assay of drug delivery system, where the drug is dispersed in the phosphate buffer. The study is with respect to in vitro and in vivo release of lovastatin. The phosphate alone appears to be a potential interfering component as observed from the present study. Several reports suggested spectrophotometric measurement
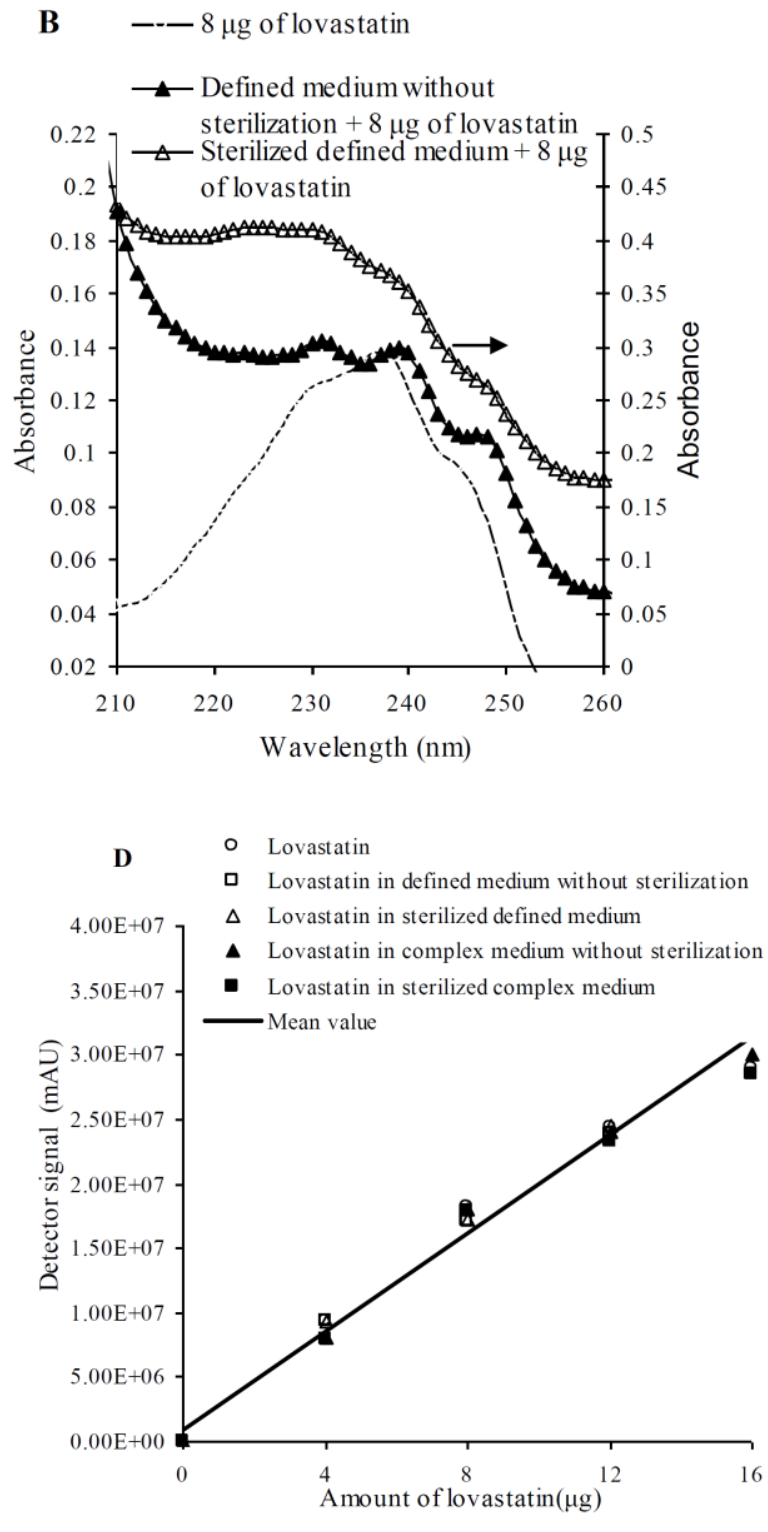

Fig. (2). Inaccuracy in lovastatin estimation by UV-visible spectrophotometry caused by phosphate and medium constituents. (A) Effect of maximum equivalent phosphate present in the medium as well as released from cells during sonication in defined medium (1.80 g/L) on the lovastatin absorption spectrum. (B) Effect of sterilization of defined medium constituents on lovastatin absorption spectrum. (C) Effect of medium constituents and phosphate on the absorbance versus amount of lovastatin plots measured by UV- visible spectrophotometry. (D) Effect of medium constituents on the standard plot of lovastatin obtained by HPLC technique. 
for those in vitro and in vivo release characteristics of the drug [29-35]. Also, a few articles reported the release characteristics of the drug in vitro by UV-visible spectrophotometry and in vivo release characteristics using HPLC analysis, considering the competitive molecules in the serum $[29,36]$.

In the development of drug delivery systems, it is important to know the stability of lovastatin and its metabolism. The lactone form of lovastatin is metabolized or converted into hydrolyzed product including the acid form of lovastatin in the liver. Thus, the hydrolyzed product concentration is more important for the drug delivery and release characteristics of the drug. This change in concentration cannot be effectively determined by UVvisible spectrophotometer [39]. Jacobson et al. (1999) [40] also measured the hydrolyzed products of lovastatin in addition to lovastatin itself by HPLC at $239 \mathrm{~nm}$. There are examples of lovastatin analogues and hydrolyzed products measured around $238 \mathrm{~nm}$ which cannot be distinguished in the spectrophotometric method.

\subsection{Effect of Medium Constituents in Lovastatin Estimation}

The constituents of the complex medium possibly contribute to error in lovastatin estimation and the corresponding absorbance values are higher than those obtained using the defined medium ( $c f$. Fig. (2B)). The sterilized complex production medium showed a higher absorbance value than the same medium before sterilization. In the case of defined production medium, autoclaving of medium increased the absorbance which resulted in errors during lovastatin estimation ( $c f$. Fig. (2B)). These results confirmed that the composition of the production medium (either defined or complex) and sterilization of the medium contribute to the error in the lovastatin assay ( $c f$. Fig. (2B) and (2C)). The magnitude of error increased with the degree of complexity of the medium and the concentration of the medium constituents. These results suggest that the spectrophotometric measurements are not suitable for quantification of lovastatin.

Pham et al. (2011) [41] reported a similar phenomenon for the estimation of xylose, where the availability of a rapid, inexpensive, and sensitive method like the spectrophotometric method is often limited by the complex nature of the medium. Some of the medium constituents, time of heating of medium, and trace elements in the medium were the reasons for inaccurate estimation of xylose.

\subsection{Quantification of Lovastatin Produced by Monascus sp. Using UV-visible Spectrophotometric and HPLC Methods}

The absorbances of aliquots of culture filtrate and of ethyl acetate-extract of culture filtrates obtained from the fermentation of Monascus purpureus and of Monascus ruber were separately measured against a suitable blank. Absorbance values were very high. There is no clear maximum peak value at $238 \mathrm{~nm}$, which probably suggest that the presence of a number of compounds could possibly contribute to the absorbance value. Results described here exclusively for Monascus purpureus.

\section{(a) Effect of Lovastatin Analogues and Its Intermediates}

Batch fermentation was carried out for lovastatin production by Monascus purpureus. The presence of lovastatin was confirmed by TLC in the fermentation broth in the entire fermentation cycle. Lovastatin was measured in the ethyl acetate extract of culture filtrate separately by HPLC technique and on a spectrophotometer. Maximum production of lovastatin was between the $6^{\text {th }}$ and $10^{\text {th }}$ day of fermentation. The absorbance read on the spectrophotometer show equivalence to statin in the $\mathrm{mg} / \mathrm{l}$ range, whereas the concentration of lovastatin measured by HPLC technique is

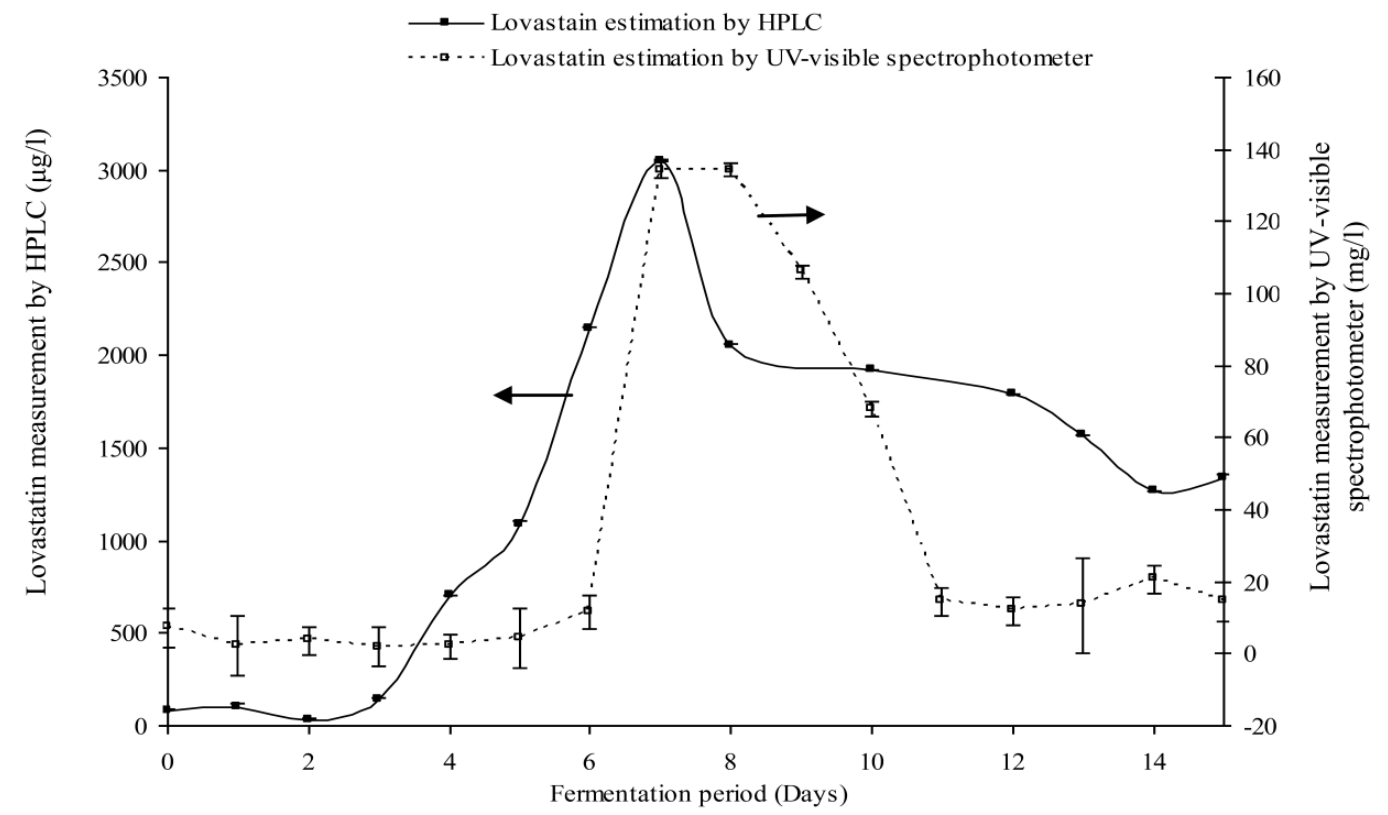

Fig. (3). Concentration profiles of lovastatin production analyzed by UV-visible spectrophotometry and HPLC. 
A

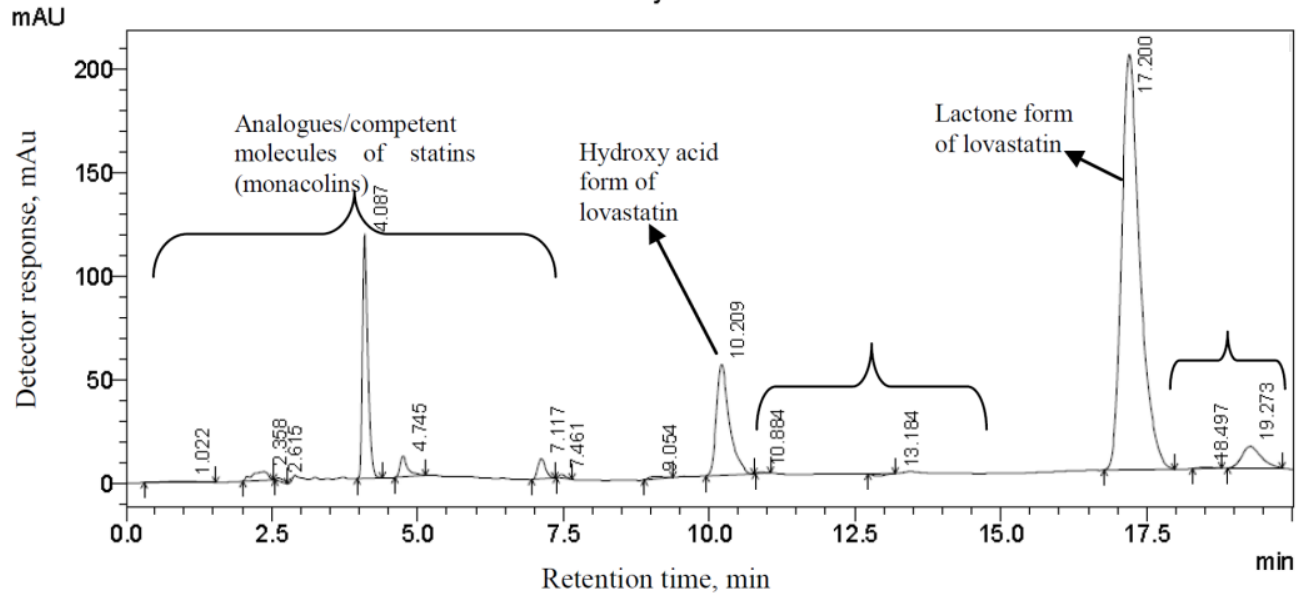

B

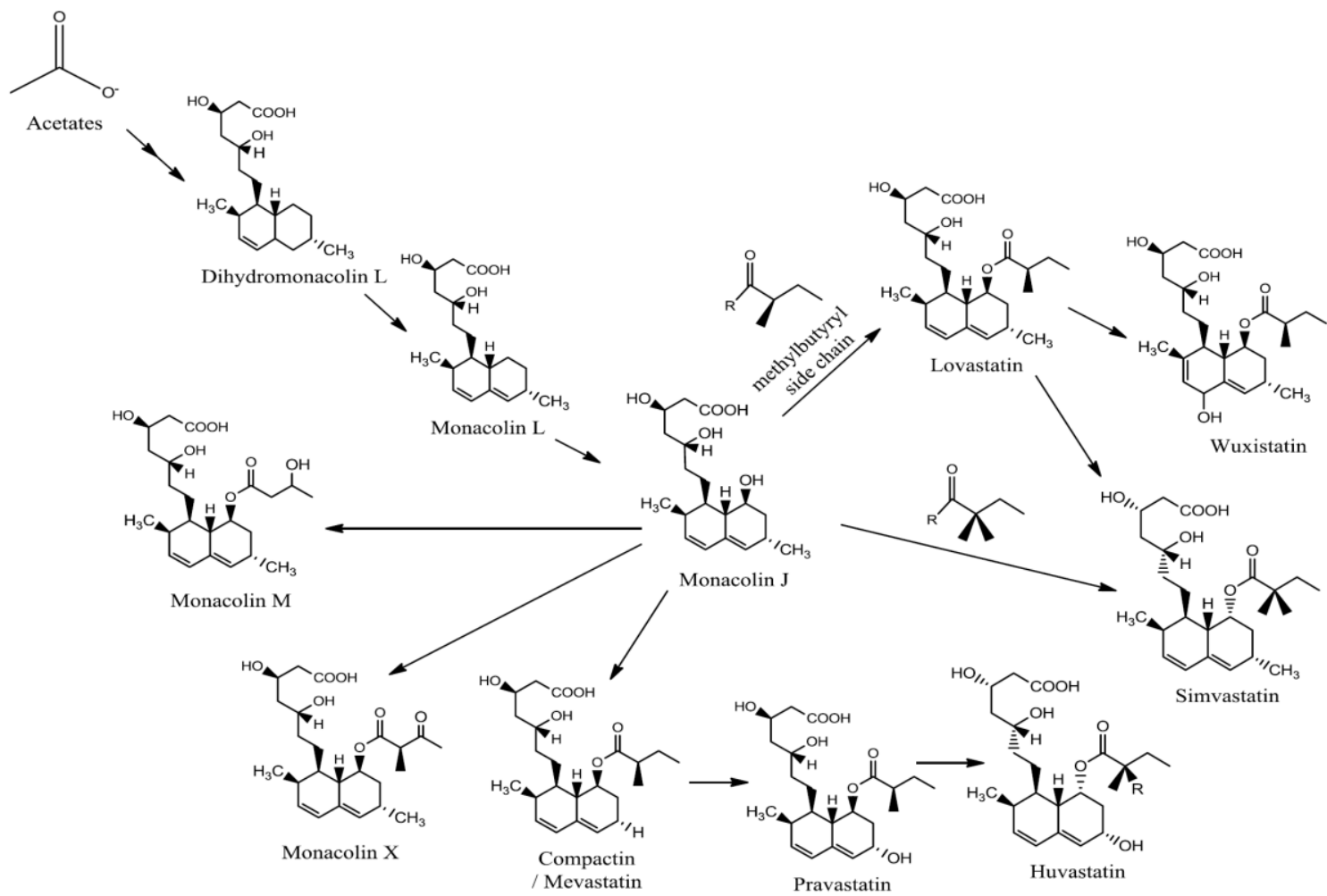

Fig. (4). Possible analogues/competent molecules present in the fermentation broth of Monascus sp. (A) Chromatogram (measured at 238 $\mathrm{nm}$ ) of Monascus purpureus fermentation samples contains individual forms of lovastatin along with its competent/analogues (monacolins). (B) Biosynthesis pathway of lovastatin and its analogues from acetate [6, 7, 44-48]. The possible compounds might be accumulated in fermentation broth, which could showed HPLC chromatogram having different analogues eluted at different rentention time. These compounds has tri-substituted heteroannular diene chromophore which absorb at $238 \mathrm{~nm}[42,44,49]$.

actually in the range of $\mu \mathrm{g} / \mathrm{l}(c f$. Fig. (3)). These results also suggest that some intermediates produced by the organism during fermentation might absorb at $238 \mathrm{~nm}$ (cf. Fig. (4)).

It has been reported that the presence of diene groups in lovastatin and some of its intermediates were responsible for this typical interference. Some of the intermediates of lovastatin, viz., monacolin $\mathrm{J}, \mathrm{X}, \mathrm{L}$, and $\mathrm{M}$ were found to have a maximum absorbance at $238 \mathrm{~nm}$ [27, 42]. In particular, monacolin $\mathrm{L}, \mathrm{J}$, and $\mathrm{X}$ were found to exhibit three maximum absorption peaks similar to the individual forms of pure lovastatin ( $c f$. Fig. 1A). However, some of the degraded compounds, viz., methyl esters, anhydro, methoxy, and acetate ester forms of lovastatin and simvastatin could absorb at $238 \mathrm{~nm}$ due to the diene groups [43]. These compounds appear as probable interferences in the quantification of lovastatin using a spectrophotometer.

Also, the pleiotropic applications of statins vary among the statins as well as the intermediates [19]. On comparison 
between the fermentation-derived statins and the synthetic statins, monacolin J (hydrolyzed product of lovastatin) show better prevention of neurodegenerative condition due to its potential blood-barrier penetration and cholesterol lowering effects on neurons [19]. Thus, the determination of analogues of statins produced in the fermentation broth is necessary, especially, when the compound is used for the preparation of nutraceuticals, fermented food etc. By the spectrophotometric method, it could not be possible to estimate the intermediates and their analogues accurately. Using spectrophotometer one can measure monacolins with relative competitive molecules at $238 \mathrm{~nm}$ ( $c f$. Fig. 4). On the other hand, HPLC system can be used to measure the intermediates along with lovastatin with greater accuracy ( $c f$. Fig. 4). It is possible that more statins could be produced including lovastatin such as compactin, pravastatin, and monacolin $\mathrm{J}$ by Monascus purpureus. This speculation is almost equivalent to the observation by Manzoni et al. [44] for Monascus sp.

Jaivel and Marimuthu determined the concentration of lovastatin by spectrophotometer, showing higher estimate for lovastatin in the range of $\mathrm{mg} / \mathrm{l}$ [9]. Studies on the applications of fermentation-derived lovastatin have used the spectrophotometric measurement for its quantification [15]. The quantification of lovastatin by spectrophotometer in the presence of intermediates also led to errors in the determination of the lethal dose concentration (LDC) in clinical studies. Osman et al. have reported the use of spectrophotometric assay method for lovastatin and found a maximum lovastatin production of $188.3 \mathrm{mg} / \mathrm{l}$ using Aspergillus terreus [10]. The high concentration of lovastatin produced could be the result of nutritional improvement of a complex medium. In this context, several reports are available for lovastatin biosynthesis using different organisms. Those reports have indicated the spectrophotometric method for the quantification of fermentationderived lovastatin [9-13].

Xie et al. reported the accumulation of monacolins especially, monacolin $\mathrm{J}$, inhibited lovastatin biosynthesis [45]. This suggests that there is a possibility of accumulation of monacolin J during the fermentation. Similarly, some of the intermediates of the lovastatin biosynthesis pathway and structural analogues of lovastatin could absorb light at 238 nm.

High absorbance values (> 0.8 after dilution) and the absence of a clear maximum at $238 \mathrm{~nm}$ could be attributed to the medium constituents, their complex nature, and the presence of secondary metabolites.

\section{CONCLUSION}

Lovastatin was estimated by UV-visible spectrophotometry and HPLC techniques. The interference of medium constituents, intermediates, and products of fermentation which led to inaccuracies during the estimation of lovastatin by spectro-photometry was discussed. The spectrophotometric method is suited for the quantification of pure lovastatin alone while HPLC analysis is the most suitable and reliable method for the estimation of lovastatin under all the experimental conditions heretofore studied.

\section{CONFLICT OF INTEREST}

The authors confirm that this article has no conflicts of interest.

\section{ACKNOWLEDGEMENTS}

The authors gratefully acknowledge the help rendered by Dr. V. Kesavan, members of Chemical Biology Laboratory, Mr. Z. Aslam Basha, and Mr. N. Arumugam of the Department of Biotechnology, IIT Madras and Mr. S. Venkatesan, Mr. S. Ravikumar, and members of Biochemical Engineering Laboratory of the Department of Chemical Engineering, IIT Madras during the different stages of this work.

\section{REFERENCES}

[1] Nigović B, Pavković I. Preconcentration of the lipid-lowering drug lovastatin at a hanging mercury drop electrode surface. J Anal Chem 2009; 64: 304-9.

[2] Abu-Nameh ESM, Shawabkeh RA, Ali A. High-performance liquid chromatographic determination of simvastatin in medical drugs. J Anal Chem 2006; 1: 63-6.

[3] Millership JS, Chin J. Determination of simvastatin in tablet formulations by derivative UV spectrophotometry. J Anal Chem 2010; 65(2): 164-8.

[4] Arayne MS, Sultana N, Hussain F, Ali SA. Validated spectrophotometric method for quantitative determination of simvastatin in pharmaceutical formulations and human serum. J Anal Chem 2007; 62: 536-41.

[5] Seenivasan A, Panda T, Théodore T. Lovastatin nanoparticle synthesis and characterization for better drug delivery. Open Biotechnol J 2011; 5: 28-32.

[6] Seenivasan A, Subhagar S, Aravindan R, Viruthagiri T. Microbial production and biomedical applications of lovastatin. Indian $\mathrm{J}$ Pharm Sci 2008; 70: 701-9.

[7] Zhuge B, Fang HY, Yu H, et al. Bioconversion of lovastatin to a novel statin by Amycolatopsis sp. Appl Microbiol Biotechnol 2008; 79: 209-16.

[8] Seenivasan A, Panda T, Théodore T. Characterization, modes of synthesis, and pleiotropic effects of hypocholesterolemic compounds-A review. Open Enzym Inhib J 2011; 4: 23-32.

[9] Jaivel N, Marimuthu P. Isolation and screening of lovastatin producing microorganisms. Int J Eng Sci Technol 2010; 2(7): 2607-11.

[10] Osman ME, Khattab OH, Zaghlol GM, Abd El-Hameed RM. Optimization of some physical and chemical factors for lovastatin productivity by local strain of Aspergillus terreus. Aust J Basic Appl Sci 2011; 5: 718-32.

[11] Sreedevi K, VenkateswaraRao J, Narasu L, Fareedullah Md. Strain improvement of Aspergillus terreus for the enhanced production of lovastatin, a HMG-COA reductase inhibitor. J Microbiol Biotech Res 2011; 1: 96-100.

[12] Latha MP, Chanakya P, Srikanth M. Lovastatin production by Aspergillus fischeri under solid state fermentation from coconut oil cake. Nepal J Biotechnol 2012; 2: 26-36.

[13] Reddy DSR, Latha PD, Latha HKPJ. Production of lovastatin by solid state fermentation by Penicillium funiculosum NCIM 1174. Drug Invention Today 2011; 3: 75-7.

[14] Prabhakar M, Lingappa K, Babu V, et al. Characterization of physical factors for optimum lovastatin production by Aspergillus terreus Klvb28mu21 under solid state fermentation. J Recent Adv Appl Sci 2012; 27: 1-5.

[15] Rajasekran A, Kalaivani M, Sabitha R. Anti-diabetic activity of aqueous extract of Monascus purpureus fermented rice in high cholesterol diet fed-streptozotocin-induced rats. Asian J Sci Inf 2009; 2: 180-9.

[16] Mielcarek J, Naskreni M, Grobelny P. Photochemical properties of simvastatin and lovastatin by radiation. J Therm Anal Calorim 2009; 96: 301-5. 
[17] Lee C-L, Wang J-J, Pan T-M. Synchronous analysis method for detection of citrinin and the lactone and acid forms of monacolin $\mathrm{K}$ in red mold rice. J AOAC Int 2006; 89: 669-77.

[18] Sayyad SA, Panda BP, Javed S, Ali M. Optimization of nutrient parameters for lovastatin production by Monascus purpureus MTCC 369 under submerged fermentation using response surface methodology. Appl Microbiol Biotechnol 2007; 73: 1054-8.

[19] Sierra S, Ramos MC, Molina P, et al. Statins as neuroprotectants: a comparative in vitro study of lipophilicity, blood-brain-barrier penetration, lowering of brain cholesterol, and decrease of neuron cell death. J Alzheimers Dis 2011; 23: 307-18.

[20] Yoshida MI, Oliveira MA, Gomes ECL et al. Thermal characterization of lovastatin in pharmaceutical formulations. J Therm Anal Calorim 2011; 106: 657-64.

[21] Li M, Fan L-Y, Zhang W, Sun J, Cao C-X. Quantitative analysis of lovastatin in capsule of Chinese medicine Monascus by capillary zone electrophoresis with UV-vis detector. J Pharm Biomed Anal 2007; 43: 387-92

[22] Damić M, Nigović B. Analysis of statins in pharmaceuticals by MEKC. Chromatographia 2010; 71: 233-40.

[23] Yang D-J, Hwang LS. Study on the conversion of three natural statins from lactone forms to their corresponding hydroxy acid forms and their determination in Pu-Erh tea. J Chromatogr A 2006; 1119: 277-84

[24] Chang Y-N, Huang J-C, Lee C-C, Shih I-L, Tzeng Y-M. Use of response surface methodology to optimize by Monascus ruber. Enzyme Microb Technol 2002; 30: 889-94.

[25] Sharaf El-Din MMK, Attia KAM, Nassar MWI, Kaddah MMY. Colorimetric determination of simvastatin and lovastatin in pure form and in pharmaceutical formulations. Spectrochim Acta A Mol Biomol Spectrosc 2010; 76: 423-8.

[26] Samiee SM, Moazami N, Haghighi S, et al. Screening of lovastatin production by filamentous fungi. Iran Biomed J 2003; 7: 29-33.

[27] Yang F, Weber TW, Gainer JL, Carta G. Synthesis of lovastatin with immobilized Candida rugosa lipase in organic solvents: effects of reaction conditions on initial rates. Biotechnol Bioeng 1997; 56: 671-80.

[28] Schachter M. Chemical, pharmacokinetic and pharmacodynamic properties of statins: an update. Fundam Clin Pharmacol 2004; 19: $117-25$.

[29] Nanjwade BK, Derkar GK, Bechra HM, Nanjwade VK, Manvi FVJ. Design and characterization of nanocrystals of lovastatin for solubility and dissolution enhancement. Nanomed Nanotechnol 2011; 2: 107. doi:10.4172/2157- 7439.1000107.

[30] Shinde AJ, More HN. Design and evaluation of polylactic-coglycolic acid nanoparticles containing simvastatin. Int J Drug Dev Res 2011; 3: 280-9.

[31] Patel MJ, Patel NM, Patel RB, Patel RP. Formulation and evaluation of self-microemulsifying drug delivery system of lovastatin. Asian J Pharm Sci 2010; 5: 266-75.

[32] Katare MK, Kohli S, Jain AP. Evaluation of dissolution enhancement of lovaststin by solid dispersion technique. Int $\mathbf{J}$ Pharm Life Sci 2011; 2: 894-8.

[33] Csempesz F, Süle A, Puskás I. Induced surface activity of supramolecular cyclodextrin-statin complexes: Relevance in drug delivery. Colloids and Surfaces A: Physicochem Eng Aspects 2010; 354: 308-13.

[34] Shinde AJ, Paithane MB, More HN. Development and in vitro evaluation of transdermal patches of lovastatin as a antilipidemic drug. Int Res J Pharm 2010; 1: 113-21.

[35] Ho M-H, Chiang C-P, Liu Y-F, et al. Highly efficient release of lovastatin from poly(lactic-co-glycolic acid) nanoparticles enhances bone repair in rats. J Orthop Res 2011; 29: 1504-10.

[36] Mandal S. Microemulsions drug delivery system: design and development for oral bioavailability enhancement of lovastatin. S Afr Pharm J 2011; 78: 44-50.

[37] Jerotskaja J, Lauri K, Tanner R, Luman M, Fridolin I. Optical dialysis adequacy sensor: wavelength dependence of the ultraviolet absorbance in the spent dialysate to the removed solutes. Conf Proc $29^{\text {th }}$ IEEE Eng Med Biol Soc 2007: pp. 2960-3.

[38] Lin F, Ma QL. A novel photoproduct of thymine in phosphatebuffered saline under far UV irradiation. Chin Chem Lett 2003; 14: 1233-5.

[39] Wang RW, Kari $\mathrm{PH}, \mathrm{Lu} \mathrm{AYH}$, et al. Biotransformation of lovastatin. IV. Identification of cytochrome P450 3A proteins as the major enzymes responsible for oxidative metabolism of lovastatin in rat and human liver microsomes. Arch Biochem Biophys 1991; 290: 355-61.

[40] Jacobsen W, Kirchner G, Hallensleben K, et al. Small intestinal metabolism of the 3-hydroxy-3-methylglutaryl-coenzyme A reductase inhibitor lovastatin and comparison with pravastatin. J Pharmacol Exp Ther 1999; 291: 131-9.

[41] Pham PJ, Hernandez R, French WT, Estill BG, Mondala AH. A spectrophotometric method for quantitative determination of xylose in fermentation medium. Biomass Bioenerg 2011; 35: 2814-21.

[42] Li Y-G, Zhang F, Wang Z-T, Hu Z-B. Identification and chemical profiling of monacolins in red yeast rice using high-performance liquid chromatography with photodiode array detector and mass spectrometry. J Pharm Biomed Anal 2004; 35: 1101-12.

[43] Krishna SR, Deshpande GR, Rao BM, Rao NS. A StabilityIndicating RP-LC method for the determination of related substances in simvastatin. J Chem Pharm Res 2010; 2: 91-9.

[44] Manzoni M, Bergomi S, Rollini M, Cavazzoni V. Production of statins by filamentous fungi. Biotechnol Lett 1999; 21: 253-7.

[45] Xie X, Watanabe K, Wojcicki WA, Wang CC, Tang Y. Biosynthesis of lovastatin analogs with a broadly specific acyltransferase. Chem Biol 2006; 13: 1161-9.

[46] Kimura K, Komagata D, Murakawa S, Endo A. Biosynthesis of monacolins: conversion of monacolin $\mathrm{J}$ to monacolin $\mathrm{K}$ (mevinolin). J Antibiot 1990; 43: 1621-2.

[47] Hutchinson CR, Kennedy J, Park C. Method of producing antihypercholesterolemic agents. US pat 6943017. 2005 Sep.

[48] Manzoni M, Rollini M. Biosynthesis and biotechnological production of statins by filamentous fungi and application of these cholesterol-lowering drugs. Appl Microbiol Biotechnol 2002; 58: 555-64.

[49] Alberts AW, Chen J, Kuron G, et al. Mevinolin: a highly potent competitive inhibitor of hydroxymethylglutaryl-coenzyme A reductase and a cholesterol-lowering agent. Proc Natl Acad Sci USA 1980; 77: 3957-61. 